\title{
Isolated Hypoglossal Nerve Palsy: A Diagnostic Challenge
}

\author{
Marina Boban ${ }^{a}$ Vesna V. Brinar ${ }^{a}$ Mario Habek ${ }^{a}$ Marko Radošb \\ University Departments of a Neurology and ${ }^{b}$ Radiology, Zagreb School of Medicine and University Hospital Center, \\ Zagreb, Croatia
}

Dear Sir,

Hypoglossal nerve palsy (HNP) is not an uncommon finding in neurological diseases [1]. However, when isolated, HNP can represent a diagnostic challenge in everyday clinical work and is limited to isolated case reports and few small case series [2]. In this article, we report 4 patients with clinical presentation of isolated HNP due to different etiologies.

\section{Case Reports}

Case 1

In August 2005, a 50-year-old Caucasian male developed constant, moderate, sharp pain which was radiating from the right mandibular angle to the right temporal region. Three days after, he experienced an abrupt onset of dysarthria as well as difficulty in moving his tongue and food boluses. He was admitted to the regional hospital with the main complaint of speech difficulties. Painful sensations lasted constantly for an additional 5 days and after that period occurred occasionally during head movements. During hospitalization brain magnetic resonance imaging (MRI) was performed. Ring-like widening of the jugular vein bulb, described on MRI, was interpreted as possible jugular vein thrombosis or paraganglioma. Findings of other tests (visual, auditory and somatosensory evoked potentials, X-rays of temporomandibular joints) were unremarkable. Three weeks after the onset of dysarthria, the patient was admitted to our hospital for an additional workup. Until then speech difficulties had diminished, but he reported occasional vague pain localized to the right mandibular angle, especially during head movements. There was no history of neck trauma or manipulation or vigorous exercise. Neurological examination revealed only right HNP with ipsilateral atrophy (fig. 1) without signs of other cranial nerve palsies as well as pyramidal signs or Horner syndrome. The patient was otherwise in excellent general condition. His medical history was unremarkable. Routine blood analysis was normal. Needle electromyography of the tongue showed denervation changes in the muscles supplied by the right hypoglossal nerve, whereas electromyographic findings in the left side of the tongue and cervical muscles were normal. Computed tomography (CT) of the brain and the base of the skull was unremarkable. In the $\mathrm{T}_{1}$-weighted MRI images without fat suppression before and after administration of gadolinium which were performed 3 weeks after the onset of symptoms, a hyperintense, round, 15mm-wide pseudoaneurysm of the right internal carotid artery was found with hypointensity in the central part of the lesion representing the residual lumen of the artery (fig. 2). The lesion extended approximately $25 \mathrm{~mm}$ distal to the right carotid artery bifurcation up to the entrance of the internal carotid artery in the carotid

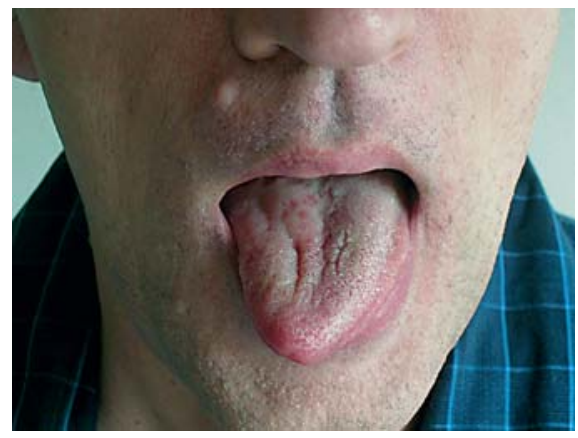

Fig. 1. Case 1. Note the right unilateral atrophy and deviation of the tongue in the patient 3 weeks after the onset of dysarthria. September 2005.

canal at the base of the right temporal bone. There were no signs of ischemic brain lesion. Additionally, CT angiography confirmed the finding of a pseudoaneurysm of the right internal carotid artery with an irregular, hyperintense, peripherally located arterial lumen (fig. 3). Digital subtraction angiography demonstrated mild asymmetrical, concentric narrowing of the right internal carotid artery in the whole length of the pseudoaneurysm. The patient recovered without anticoagulation therapy. At the follow-up, 1 month later, further improvement in tongue movements was observed.

\section{KARGER}

Fax +4161306 1234 E-Mail karger@karger.ch www.karger.com
(C) 2007 S. Karger AG, Base 0014-3022/07/0583-0177\$23.50/0 www.karger.com/ene
Marina Boban

University Department of Neurology

Zagreb School of Medicine and University Hospital Cente

Kišpatićeva 12, HR-10000 Zagreb (Croatia)

Tel. +38591539 5594, Fax +3851238 8045, E-Mail maboban@mef.hr 


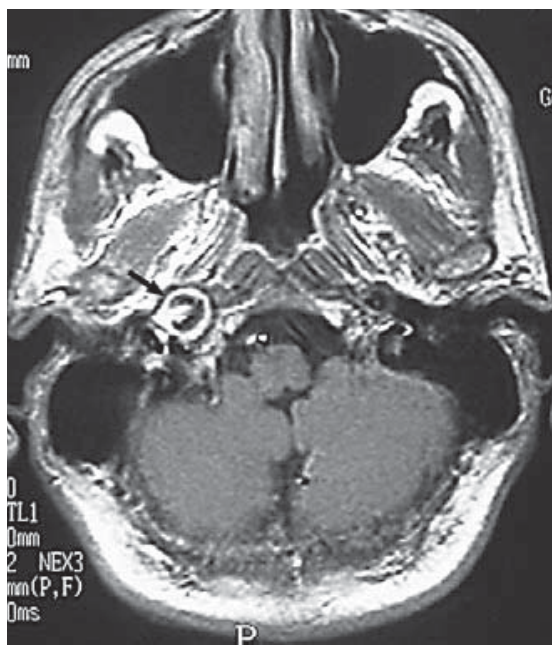

Fig. 2. Case $1 . T_{1}$-weighted MRI image without fat suppression performed 3 weeks after the onset of symptoms revealed a hyperintense, round, 15-mm-wide pseudoaneurysm of the right internal carotid artery with hypointensity in the central part of the lesion (arrow) representing the residual lumen of the artery. September 2005.

Case 2

A 61-year-old Caucasian female was admitted in August 2005 to our emergency room with a severe, diffuse, sharp headache, which had started insidiously 10 days previously and was refractory to medical treatment. During neurological examination, positive meningeal signs were found together with right HNP and ipsilateral tongue atrophy, without speech difficulties. Brain CT was unremarkable and the cerebrospinal fluid (CSF) finding was not consistent with subarachnoid hemorrhage. On contrast-enhanced CT scan, a suspect, expansive formation beneath the base of the skull was revealed located between the jugular foramen and foramen magnum and destroying the right occipital condyle and clivus (fig. 4). In brain $\mathrm{MRI} \mathrm{T}_{1}$-weighted images, an extracranial, partially necrotic expansive lesion located beneath the right occipital condyle and hypoglossal canal aperture with destruction of the clivus and anterior arch of the atlas was revealed (fig. 5). On digital subtraction angiography, 2 aneurysms were found, on the right and left middle cerebral artery each. There were no signs of arterial-venous malformations or pathological vascularization of the suspected expansive lesion. Finally, ovarian cancer was found to be the primary tu-
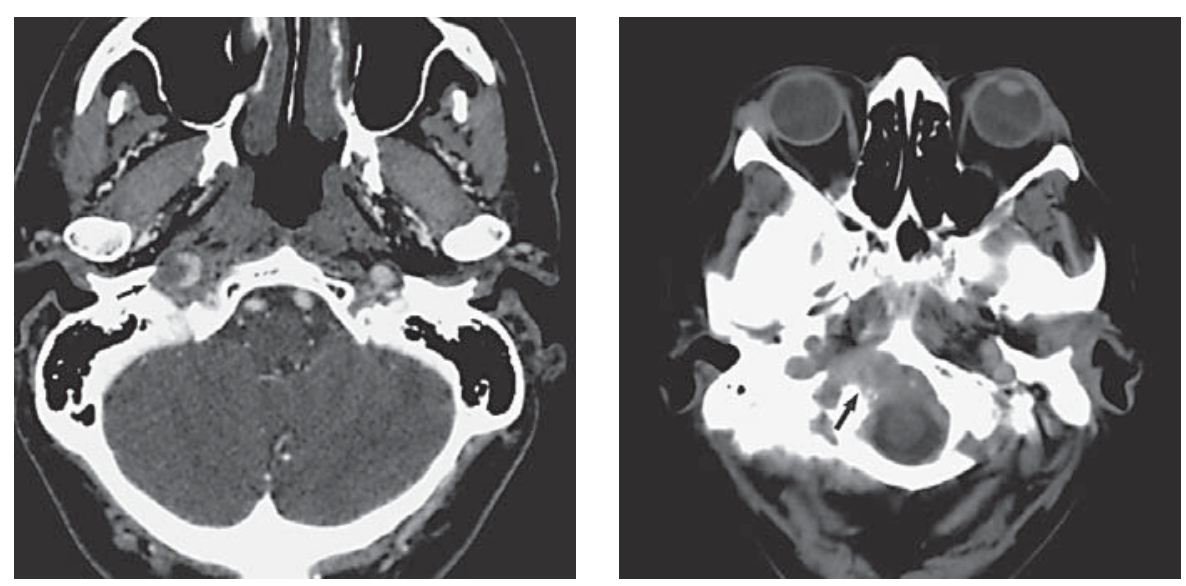

Fig. 3. Case 1. CT angiography confirmed the finding of a pseudoaneurysm of the right internal carotid artery (arrow) with an irregular, hyperintense, peripherally located arterial lumen. September 2005.

mor with additional liver and lung metastases as well as metastasis of the base of the skull, which caused the isolated HNP.

\section{Case 3}

In March 1999, a 15-year-old girl had abruptly developed nausea, vomiting and vertiginous symptoms after an episode of a nonspecific upper respiratory tract infection. The symptoms lasted for several days and then completely disappeared. A few weeks later she became slightly ataxic and noted some atrophy of the right side of the tongue. Neurological examination revealed right unilateral atrophy and deviation of the tongue upon protrusion. Large lesions, hyperintense on fluid-attenuated inversion recovery and $\mathrm{T}_{2}$-weighted MRI images and hypointense on $\mathrm{T}_{1}$-weighted MRI images without fat suppression, located periventriculary in the right frontal lobe, right cerebellar hemisphere as well as medulla oblongata were revealed (fig. 6). CSF examination revealed positive oligoclonal bands. Immunological tests were normal, and serum Epstein-Barr virus antibodies were elevated, indicating past infection. After pulse corticosteroid treatment, all symptoms resolved except atrophy of the right half of the tongue. She was diagnosed as having postinfectious acute disseminated
Fig. 4. Case 2. Contrast-enhanced CT scan demonstrated a suspect, expansive formalocated between the jugular foramen and foramen magnum and destroying the right occipital condyle and clivus. September 2005 . tion beneath the base of the skull (arrow)

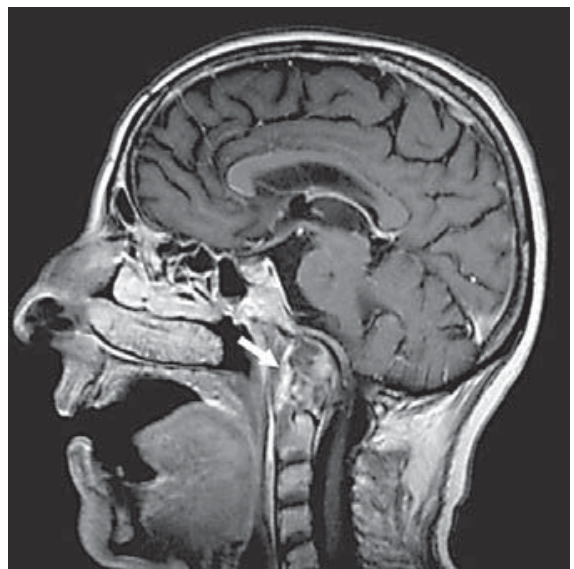

Fig. 5. Case 2. Brain $\mathrm{T}_{1}$-weighted MRI revealed an extracranial, partially necrotic expansive lesion (arrow) located beneath the right occipital condyle and hypoglossal canal aperture with destruction of the clivus and anterior arch of the atlas. August 2005 .

encephalomyelitis and treated with pulse corticosteroid therapy. On control visits in 2002, 2003 and 2005 she was free of symptoms, but severe right unilateral atrophy and deviation of the tongue persisted. 


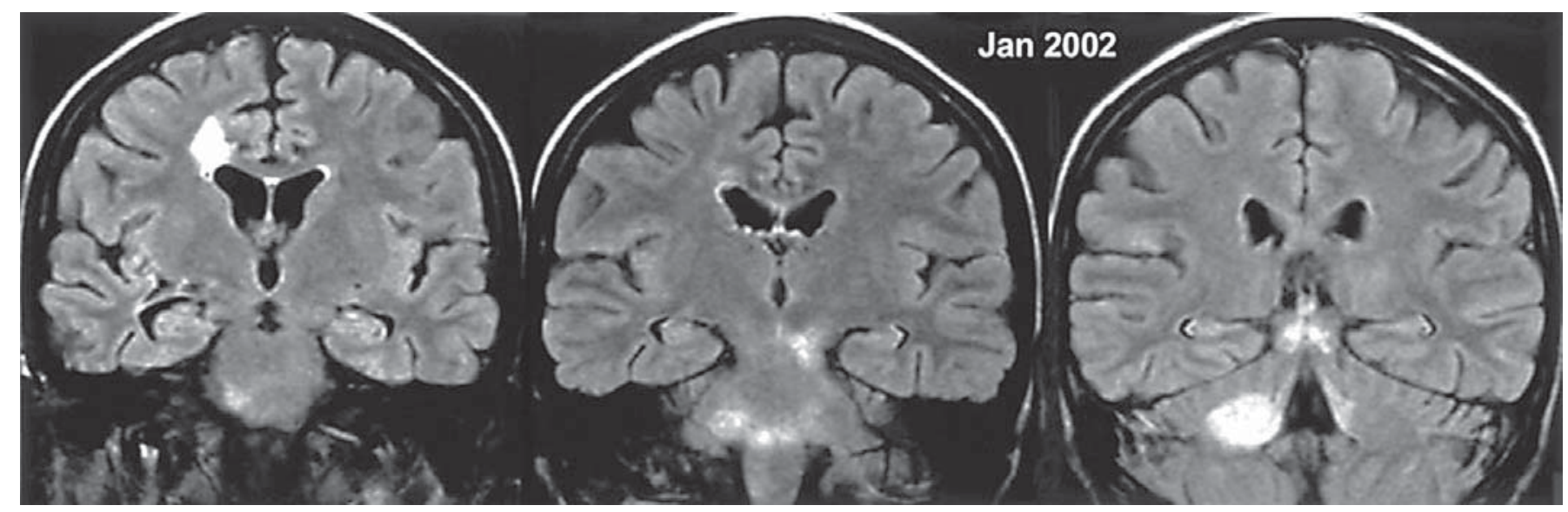

Fig. 6. Case 3. Fluid-attenuated inversion recovery MRI demonstrated large hyperintense lesions located periventriculary in the right frontal lobe and in the right cerebellar hemisphere, as well as in the medulla oblongata. January 2002.

Case 4

In September 2002, a 63-year-old female presented to our emergency room because of severe, sharp pain most prominent in the right, cervico-occipital region with spreading through the ipsilateral mandibular and temporal regions. The pain was refractory to medical treatment. The patient also noticed problems with speech. Beside mild dysarthria due to a slight right deviation of the tongue following protrusion, the remainder of the neurological examination was normal. Her medical history revealed hyperthyreosis of 20 years ago. In 1994 infiltrative ductal cancer of the right breast had been diagnosed with metastases in the ipsilateral axilla. Surgical procedures followed by irradiation and chemotherapy were performed. In May 2001, she was reoperated and started taking tamoxifen. Routine blood analysis, serum cancer markers, repeated CSF findings as well as findings of brain CT were unremarkable. In the $\mathrm{T}_{2}$ weighted images, an expansive lesion was revealed located on the right ventrolateral surface of the medulla oblongata (fig. 7). The lesion was completely excised with histologically confirmed breast cancer metastasis. Finally, adjuvant radiotherapy was performed.

\section{Discussion}

The hypoglossal nerve is a pure motor nerve that innervates intrinsic as well as extrinsic muscles of the tongue. It could be divided into 5 segments: medullary (nu-

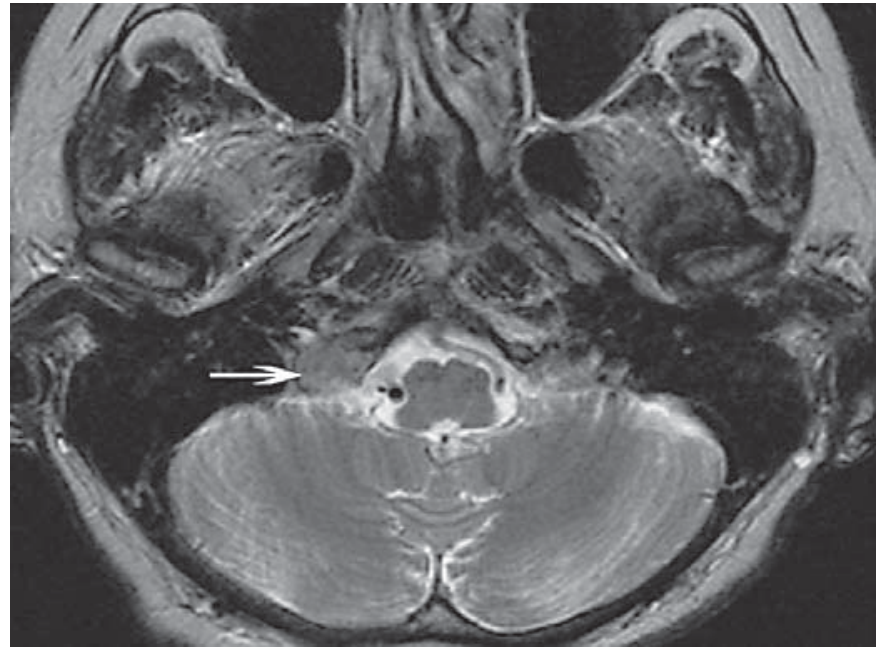

Fig. 7. Case 4. Brain $\mathrm{T}_{2}$-weighted MRI revealed an expansive lesion (arrow) located on the right ventrolateral surface of the medulla oblongata. September 2002.

clear), cisternal (extramedullary intracranial), skull base (the segment which passes through the hypoglossal nerve canal), nasopharyngeal, oropharyngeal and carotid (in close vicinity to the glossopharyngeal and vagus nerves as well as to the internal carotid artery), and sublingual space (where its branches terminate innervating lingual muscles). The nerve can be damaged anywhere during its course. Localizing the lesion could allow the clinician to narrow possible etiological factors.
Occurrence of HNP accompanied with other cranial nerve palsies and long tract signs is not an uncommon finding. In a retrospective analysis of $100 \mathrm{HNPs}$, Keane [1] found that tumors, predominantly malignant, were the most common cause of HNP (49 cases), whereas other causes were trauma, medullary infarctions, multiple sclerosis, Guillain-Barré neuropathy and infection. However, isolated HNP is a rare finding, because of its proximity to other important anatomical structures through- 
out its pathway, and is described in several case reports [3-23] and a very small case series [2]. Combarros et al. [2] reported 9 patients with isolated HNP caused by tumors, Chiari malformation and dural arteriovenous fistula, whereas, in 4 patients, who had an excellent outcome, no cause was found. Isolated HNP represents a diagnostic challenge in everyday clinical work. Even today, a great number of these patients are misdiagnosed. Therefore, finding the appropriate diagnostic tool and knowledge of hypoglossal nerve anatomy is of major importance.

Isolated HNP is rarely caused by internal carotid artery dissection (ICAD). Direct compression of the nerve in the parapharyngeal space by a pseudoaneurysm or compression of small nutrient vessels supplying the nerve could lead to the palsy. ICAD is in its typical presentation, which is found in less that one third of patients, accompanied with pain in the head, neck or face ipsilateral to the dissected artery, accompanied by a partial Horner's syndrome and followed hours or days later by cerebral or retinal ischemia [24]. Therefore, the presence of typical pain with cranial nerve palsy (which can be present in $12 \%$ of patients [25]) should strongly suggest the diagnosis. In a diagnostic approach, MRI has a crucial role. MR angiography, CT angiography or digital subtraction angiography might be good additional tests. In case 1 , the patient was, according to the first brain MRI, misdiagnosed as having paraganglioma in the area of the jugular vein bulb. Persistent pain in the temporal region implicated the necessity of MRI revision, which revealed a right ICAD. The diagnosis was additionally confirmed with CT angiography. Since the patient was admitted to our hospital in the chronic phase of ICAD and based on the recent guidelines for ICAD treatment [26], we did not start anticoagulation or antithrombotic therapy. During the follow-up period, his condition improved. The clinical presentation of ICAD with isolated HNP and without evidence of Horner's syndrome as well as other cranial nerve palsy or cerebral ischemia is quite a rare and unusual finding.

Tumors of the base of the skull are the most frequent cause of isolated HNP $[1,2]$ with nasopharyngeal and metastatic carcinoma as the most common examples [1]. Metastases originate mostly from breast, renal cell and prostate cancer. In case 2, besides isolated HNP, the patient present- ed with severe, 10-day-lasting, diffuse headache refractory to medical treatment with additional positive meningeal signs. According to the symptoms and findings in this patient, subarachnoidal hemorrhage had to be ruled out. Although, on CT angiography and DSA, 2 aneurysms were revealed, lumbar puncture was unremarkable. Finally, using CT of the base of the skull and additional MRI, an expansive lesion in the region of the hypoglossal nerve canal was found. The positive meningeal signs in this case were due to destruction of the first cervical vertebrae and clivus. To our best knowledge, there have been no reports of isolated HNP caused by ovarian cancer so far. In addition, in case 4 , the patient presented with severe pain localized mostly in the right cervico-occipital region with right hypoglossal nerve involvement. Although leptomeningeal carcinomatosis was suspected, repeated CSF examination did not reveal malignant cells. Finally, brain MRI revealed an expansive lesion in the region of the cisternal segment of the right hypoglossal nerve which was due to breast cancer metastasis.

Isolated cranial nerve palsies are rare clinical findings in multiple sclerosis and when present are usually limited to the third, fourth, sixth, seventh and eighth cranial nerves [27]. When HNP is involved, it is due to demyelination in the medulla, at the level of the hypoglossal motor nuclei where nerve roots leave the ventral side of the nuclei and go to the reticular formation. Keane [1] in his series mentioned 5 patients with clinically definitive multiple sclerosis; however, there were no further details on clinical presentation and whether associated symptoms or signs were present or not. Even sparser are data on cranial nerve involvement in acute disseminated encephalomyelitis. There are reports, mainly in children, describing postinfectious HNP, usually after Epstein-Barr virus infection $[15,16]$. Additionally, hypoglossal affection was reported in Behçet's disease where it represents a form of mononeuritis [19]. Our patient seems to be the first described in the literature with a perinuclear HNP as a consequence of acute disseminated encephalomyelitis.

In conclusion, HNP, when isolated, represents a challenge in everyday clinical work. A thorough patient history accompanied with good knowledge of hypoglossal nerve anatomy as well as a rational selection of diagnostic tests is necessary for making the diagnosis. We suggest MRI as the diagnostic procedure of first choice to localize the lesion in patients with suspected ICAD. MR angiography, CT angiography or digital subtraction angiography might be good additional tests. In cases of suspected expansive lesions, CT of the base of the skull could be a sufficient initial procedure. However, if negative, MRI focusing on the hypoglossal nerve pathway should be obtained. Brain MRI and additional workup (such as CSF finding and visual evoked potentials) are needed in case of suspected demyelinating disease.

\section{References}

1 Keane J: Twelfth-nerve palsy: analysis of 100 cases. Arch Neurol 1996;53:561-566.

2 Combarros O, Alvarez de Arcaya A, Berciano J: Isolated unilateral hypoglossal nerve palsy: nine cases. J Neurol 1998;245:98100.

3 Passacantilli E, Lanzino G, Henn JS, Partovi S, Spetzler RF: Intracranial extradural schwannoma of the 12th cranial nerve: case illustration. J Neurosurg 2003;98:219.

4 Barnadas MA, Escartin A, Alomar A: Oral ulcerations as the first sign of a foramen magnum meningioma. Oral Surg Oral Med Oral Pathol Oral Radiol Endod 2000;90: 609-611.

5 Tucker D, Musuka C: Isolated 12th cranial nerve palsy as a complication of multiple myeloma. Br J Haematol 2004;124:1.

6 Naidich TP, Pudlowski RM, Leeds NE, Deck MD: Case report - Hypoglossal palsy: computed tomography demonstration of denervation hemiatrophy of the tongue associated with glomus jugulare tumor. J Comput Assist Tomogr 1978;2:630-632.

7 Silvester KC, Barnes S: Adenoid cystic carcinoma of the tongue presenting as a hypoglossal nerve palsy. Br J Oral Maxillofac Surg 1990;28:122-124.

8 Tommasi-Davenas C, Vighetto A, Confavreux C, Aimard G: Causes of paralysis of the hypoglossal nerve: a propos of 32 cases. Presse Méd 1990;19:864-868.

9 Singh S, Shankar RR, Singhi PD, Kumar L: Isolated hypoglossal nerve palsy in pyogenic meningitis. Indian Pediatr 1988;25:705706.

10 Cheng VST, Schultz MD: Unilateral hypoglossal nerve atrophy as a late complication of radiation therapy of head and neck carcinoma: a report of four cases and a review of the literature on peripheral and cranial nerve damages after radiation therapy. Cancer 1975;35:1537-1544.

11 Spitzer C, Mull M, Topper R: Isolated hypoglossal nerve palsy caused by carotid artery dissection: the necessity of MRI for diagnosis. J Neurol 2001;248:909-910. 
12 Sturzenegger M, Huber P: Cranial nerve palsies in spontaneous carotid artery dissection. J Neurol Neurosurg Psychiatry 1993; 56:1191-1199.

13 McKeon A, Murphy S, McNamara B, Ryder $\mathrm{DQ}$, Galvin RJ: Isolated hypoglossal nerve palsy due to compression by a dissecting vertebral artery. Eur Neurol 2005;53:162-164.

14 Adams H, Verhoff MA, Hagemeyer TP, Muller KM: Arachnoid cyst with consecutive brain stem atrophy, hypoglossal nerve paresis and tongue atrophy. Pathologe 2001; 22:266-269.

15 Parano E, Giuffrida S, Restivo D, Saponara R, Greco F, Trifiletti RR: Reversible palsy of the hypoglossal nerve complicating infectious mononucleosis in a young child. Neuropediatrics 1998;29:46-47.

16 Wright GD, Lee KD: An isolated right hypoglossal nerve palsy in association with infectious mononucleosis. Postgrad Med J 1980; 56:185-186.
17 DeSimone PA, Snyder D: Hypoglossal nerve palsy in infectious mononucleosis. Neurology 1978;28:844-847.

18 Blankenship LD, Basford JR, Strommen JA, Andersen RJ: Hypoglossal nerve palsy from cervical spine involvement in rheumatoid arthritis: 3 case reports. Arch Phys Med Rehabil 2002;83:269-272.

19 Ko KF, Kwan MC, Chan TP, Kay CS, Lam EK: Isolated hypoglossal nerve palsy in Behçet's disease. Ann Acad Med Singapore 1997;26:848-849.

20 Hadjikoutis S, Jayawant S, Stoodley N: Isolated hypoglossal nerve palsy in a 14-yearold girl. Eur J Paediatr Neurol 2002;6:225228.

21 Felix JK, Schwartz RH, Myers GJ: Isolated hypoglossal nerve paralysis following influenza vaccination. Am J Dis Child 1976;130: $82-83$.
22 Giuffrida S, Lo Bartolo ML, Nicoletti A, et al: Isolated, unilateral, reversible palsy of the hypoglossal nerve. Eur J Neurol 2000;7:347349.

23 Sugama S, Matsunaga T, Ito F, Eto Y, Maekawa K: Transient, unilateral, isolated hypoglossal nerve palsy. Brain Dev 1992;14:122123.

24 Schievink WI: Spontaneous dissection of the carotid and vertebral arteries. N Engl J Med 2001;344:898-906.

25 Mokri B, Silbert PL, Schievink WI, Piepgras DG: Cranial nerve palsy in spontaneous dissection of the extracranial internal carotid artery. Neurology 1996:356-359.

26 Lyrer P, Engelter S: Antithrombotic drugs for carotid artery dissection. Stroke 2004;35: 613-614.

27 Thomke F, Lensch E, Ringel K, Hopf HC: Isolated cranial nerve palsies in multiple sclerosis. J Neurol Neurosurg Psychiatry 1997;63:682-685. 